
doi:10.1017/S0033291711000602

\title{
Daily cortisol, stress reactivity and psychotic experiences in individuals at above average genetic risk for psychosis
}

\author{
D. Collip ${ }^{1}$, N. A. Nicolson ${ }^{1} \dagger$, M. Lardinois ${ }^{1} \dagger$, T. Lataster ${ }^{1}$, J. van Os $^{1,2}$ and I. Myin-Germeys ${ }^{1 *}$ \\ for G.R.O.U.P.t \\ ${ }^{1}$ Department of Psychiatry and Neuropsychology, South Limburg Mental Health Research and Teaching Network, EURON, \\ Maastricht University Medical Centre, Maastricht, The Netherlands \\ ${ }^{2}$ King's College London, King's Health Partners, Department of Psychosis Studies, Institute of Psychiatry, London, UK
}

\begin{abstract}
Background. Hypothalamic-pituitary-adrenocortical (HPA) axis abnormalities have been found in patients with a psychotic disorder and first-degree relatives of patients with a psychotic disorder react with subtle increases in non-clinical psychotic experiences and negative emotions in the face of everyday stress. The current study investigated whether HPA axis functioning is altered in individuals at above average genetic risk for psychotic disorder, examining diurnal cortisol profiles, cortisol reactivity to daily stressors and the association between HPA axis activity and subclinical psychotic experiences.
\end{abstract}

Method. Participants included siblings of patients with a psychotic disorder $(n=60)$ and a healthy comparison group $(n=63)$. The Experience Sampling Method (a structured diary technique) was employed to assess stress, psychotic experiences, negative affect and salivary cortisol repeatedly in the flow of daily life.

Results. Multi-level analyses revealed higher diurnal cortisol levels and heightened cortisol reactivity to negative daily events in siblings compared with controls. Diurnal cortisol slope did not differ between the two groups, but momentary increases in psychotic experiences and negative affect were associated with increased cortisol in the sibling group.

Conclusions. Findings support altered HPA axis activity in individuals at above average genetic risk for psychotic disorder, as evidenced by higher diurnal cortisol levels and increased cortisol reactivity to daily stress. Results also suggest a dynamic association between cortisol secretion and the intensity of psychotic-like experiences and negative emotions in daily life, although the direction of this association remains to be elucidated.

Received 18 November 2010; Revised 28 March 2011; Accepted 29 March 2011

Key words : Cortisol, daily stress, HPA axis, momentary assessment, negative affect, psychosis, siblings.

\section{Introduction}

Minor stresses in the flow of daily life have repeatedly been associated with increases in psychotic symptoms and negative emotions in individuals with a psychotic disorder (Myin-Germeys et al. 2005a). However, the biological substrate underlying this phenomenon remains unknown and vulnerability markers need to be identified. Results of experimental studies suggest that increased psychotic reactivity to stress may reflect increased dopamine reactivity (Myin-Germeys et al.

\footnotetext{
* Address for correspondence: Prof. Dr. I. Myin-Germeys, Department of Psychiatry and Neuropsychology, Maastricht University, PO Box 616 (VIJV1), 6200 MD Maastricht, The Netherlands.

(Email: i.germeys@maastrichtuniversity.nl)

$\dagger$ These authors contributed equally to this work.

\$ G.R.O.U.P. members are listed in the Appendix.
}

2005b; Soliman et al. 2008). In addition, several lines of evidence indicate that dysregulation of the hypothalamic-pituitary-adrenocortical (HPA) axis may play a role in the relationship between stress and psychotic experiences (van Winkel et al. 2008; Walker et al. 2008). In individuals with a psychotic disorder, abnormalities have been found at various levels of the HPA axis, including functional and structural changes in the hypothalamus (Koolschijn et al. 2008), the pituitary (Pariante, 2008) and the hippocampus (Wright et al. 2000). Similarly, cortisol levels and reactivity to stress may be disturbed (Albus et al. 1982; Breier et al. 1988; Jansen et al. 1998; Jansen et al. 2000; Marcelis et al. 2004; Ryan et al. 2004; Walsh et al. 2005; Brenner et al. 2009; Mondelli et al. 2010). Nevertheless, it remains uncertain whether HPA axis abnormalities are causally related to the increased emotional and psychotic stress reactivity observed in patients with a psychotic disorder. 
One of the major obstacles to research in this area is that patients with a psychotic disorder usually receive antipsychotic medication, which can affect cortisol levels and brain structures (Meltzer et al. 1989; Wik, 1995; Pariante, 2008). Thus, studies in patient samples cannot yield firm conclusions about HPA axis dysregulation as a biological substrate or vulnerability marker. Studies in patients with a first episode of psychotic illness are informative in that they are not confounded by long-term medication or illness effects. However, they cannot establish the degree to which increased HPA axis activity constitutes a precursor of psychosis, as reflected in an increased vulnerability to stress, as opposed to being the result of the psychosis and related stress. Therefore, studies have been conducted in persons who are prone to psychosis but have not developed a psychotic disorder. Schizotypal adolescents, for example, were found to have higher cortisol levels than controls (Mittal et al. 2007) and heightened cortisol secretion in another sample of schizotypal adolescents was associated with schizotypal symptomatology at 2-year follow-up (Walker et al. 2001). The potential predictive value of HPA axis abnormalities is further supported by findings in a sample of young people at ultra-high risk of psychotic disorder; months before onset, those who later developed a psychotic disorder had larger pituitary volumes than those who did not develop a disorder (Garner et al. 2005). Walker et al. (2010) recently reported that higher cortisol levels predicted higher risk of conversion to psychotic disorder in an at-risk sample studied longitudinally. Together, these findings add support to the hypothesis that heightened stress reactivity may be one of the risk factors underlying both HPA axis abnormalities and vulnerability to psychotic disorder.

Healthy siblings of individuals with a psychotic disorder are another important research population: they share not only genetic vulnerability for psychosis with their affected siblings, but also many sociodemographic, parental and developmental characteristics. First-degree relatives show increases in the intensity of subtle psychotic experiences and negative emotions in reaction to daily hassles (Myin-Germeys et al. 2001b , 2005a). Moreover, studies have reported structural HPA axis abnormalities, such as increased pituitary (Mondelli et al. 2008) and hypothalamic volumes (Goldstein et al. 2007), in relatives of patients with schizophrenia. However, sibling studies have not yet examined cortisol secretory patterns or the possible association of cortisol with increased emotional and psychotic reactivity to stress in daily life.

The current study, therefore, assessed cortisol in a sample of siblings of patients with psychotic disorder and matched controls. We used the Experience
Sampling Method (ESM), a structured diary technique, to assess daily hassles, psychotic experiences and negative emotions at frequent intervals over the course of several days, with concurrent sampling of salivary cortisol. In studies conducted in the natural environment, cortisol is generally characterized in terms of overall levels, diurnal patterns and reactivity to acute stressors (Nicolson, 2007). Cortisol has a pronounced circadian rhythm, with a sharp increase in the first hour after awakening and a gradual decrease over the rest of the day. Acute stressors can induce short-term peaks, with a half-life of approximately $1 \mathrm{~h}$. The design of the current study allowed investigation of several of these aspects of cortisol secretion. The aims were threefold: (i) to examine differences in overall cortisol levels and diurnal slope between siblings and controls; (ii) to investigate differences between siblings and controls in cortisol reactivity in response to naturally occurring stressors; (iii) to determine the association between momentary psychotic experiences or negative emotions and momentary cortisol in siblings compared with controls. In case of significant group differences, we performed additional analyses to explore alternative (non-genetic) explanations of any difference in cortisol patterns (including effects of depression, childhood trauma, current stress and distress levels and degree of psychometric psychosis liability).

\section{Method \\ Participants}

The sample consisted of 71 healthy siblings (68 full siblings and three half-siblings) of patients diagnosed with a non-affective psychotic disorder and 66 control subjects. The siblings were recruited through their ill relatives or family support organizations as part of a larger study (G.R.O.U.P., 2010). Controls were selected through random mailings to addresses in the residential areas of patients and siblings. Written informed consent, conforming to local ethics committee guidelines, was obtained from all subjects. The Comprehensive Assessment of Symptoms and History (Andreasen et al. 1992) was completed to assess symptom history over the past 6 months and lifetime, yielding DSM-IV diagnoses (APA, 1994). Inclusion criteria were: (i) age 16-55 years; (ii) sufficient command of the Dutch language. Exclusion criteria were: (i) use of steroid medication; (ii) current Axis 1 disorder; (iii) lifetime history of psychotic disorder; and, for the controls, (iv) family history of psychotic disorder as assessed by the FIGS (NIMH Genetics Initiative, 1992). 


\section{ESM}

The ESM is a random time-sampling self-assessment technique; studies have demonstrated the feasibility, validity and reliability of ESM in general and patient populations (Csikszentmihalyi \& Larson, 1987; MyinGermeys et al. 2009). Subjects received a digital wristwatch that emitted a signal 10 times per day on six consecutive days, at unpredictable moments between 07:30 and 22:30 hours. After each 'beep', subjects completed ESM self-assessment forms concerning current context, thoughts, emotions and psychotic experiences. Subjects were instructed to complete their reports immediately after the beep, thus minimizing memory distortions. Reports were considered valid when subjects responded within 15 min after the beep, as determined by comparing the actual beep time with the reported time of completion. For inclusion in the analyses, participants had to have provided valid responses to at least one-third of the emitted beeps (Delespaul et al. 2002).

\section{Salivary cortisol sampling}

After each ESM beep, subjects collected a saliva sample with a cotton swab (Salivette; Sarstedt, The Netherlands), replaced the swab in the salivette tube, and recorded the exact collection time. Samples were stored in subjects' home freezers until transport to the laboratory, where uncentrifuged samples were kept at $-20^{\circ} \mathrm{C}$ until analysis. Saliva samples collected more than $15 \mathrm{~min}$ after the beep were excluded from the analysis.

\section{ESM measures}

Event stress

Stress was conceptualized in terms of subjective appraisals of events and minor disturbances that continually occur in the natural flow of daily life. After each beep, participants were asked to report the most important event that had happened between the current and the previous report and then to rate this event on a 7-point, bipolar Likert scale $(-3=$ very unpleasant, $0=$ neutral, $3=$ very pleasant). For the current analyses, all positive responses were recoded as 0 and the negative responses were recoded so that high scores reflect more unpleasant and potentially stressful events $(0=$ neutral, $3=$ very unpleasant) (Jacobs et al. 2007).

\section{Psychotic experiences}

Psychotic symptomatology was assessed with eight ESM items rated on 7 -point Likert scales $(1=$ not at all to $7=$ very): 'My thoughts are now paranoid', 'My thoughts are difficult to express', 'I can't get rid of my thoughts', 'My thoughts are influenced by other people', 'I feel unreal', 'I hear voices', 'I see things' and 'I am afraid of losing control' (Myin-Germeys et al. 2005a). The mean of these eight ratings formed the variable psychotic experiences.

\section{Negative affect}

Negative affect was assessed as the mean score on five ESM items, rated on 7-point Likert scales $(1=$ not at all to $7=$ very): 'I feel insecure', 'I feel lonely', 'I feel anxious', 'I feel down' and 'I feel guilty'.

\section{Salivary cortisol}

Salivary cortisol is a reliable and non-invasive measure of the free, unbound cortisol in blood, which is considered to be the biologically active hormone. Radio-immunoassays were run in duplicate, using a tracer solution of cortisol-3CMO coupled with $2-\left[{ }^{125} \mathrm{I}\right]$ histamine and specific antibodies raised against cortisol-3CMO-BSA (Sulon et al. 1978) (Dr J. Sulon, University of Liège). The lower detection limit of the assay was $0.2 \mathrm{nmol} / 1$. The intra- and inter-assay coefficients of variation were $<5 \%$ and $<12 \%$, respectively. All samples from an individual were analysed in the same assay to reduce sources of variability. Observations with cortisol $>44 \mathrm{nmol} / 1(n=11)$ were considered physiologically abnormal and were excluded from the statistical analysis (van Eck et al. 1996; Peeters et al. 2004; Jacobs et al. 2007).

\section{Questionnaire measures}

Trait psychosis liability

Participants completed the 40-item Community Assessment of Psychic Experiences (CAPE) (Hanssen et al. 2005), a self-report instrument that captures variation in the positive and negative dimensions of non-clinical psychotic experiences as well as variation in depression. The total score on the positive dimension (hereafter CAPE trait score) was used as indicator of psychometric psychosis liability.

\section{Childhood trauma}

Participants completed a Dutch version of the Childhood Trauma Questionnaire 25-item short form (CTQ) (Bernstein et al. 2003). The CTQ consisted of 25 questions rated on a 5-point Likert scale enquiring about traumatic experiences in childhood. A general measure of childhood trauma was generated 
by calculating the sum of the answers to all 25 questions.

\section{Statistical analyses}

Multi-level modelling approach

Analyses of variance and $\chi^{2}$ tests were conducted to investigate group differences in demographic characteristics, cross-sectional measures and mean ratings on ESM variables. ESM and cortisol data were analysed using multi-level regression techniques, which take the hierarchical structure of the data into account. Altogether, 35 families provided more than one participant, resulting in a further level of clustering. Thus, in the current study, repeated momentary measurements (level 1) were clustered within days (level 2), within subjects (level 3), some of whom were members of the same family (level 4). Data were analysed using the XTMIXED multi-level random regression routine in Stata 11.0 (StataCorp, 2009). The $\beta$ 's are the fixed regression coefficients of the predictors in the multi-level model. When significant interactions were found, stratified analyses were conducted to clarify group differences, using the STATA LINCOM command to calculate the appropriate linear combinations.

Cortisol values were $\log$ transformed to reduce skewness of distribution, generating the variable lncort. The variable time was centred around the grand mean for all samples. To model the cortisol diurnal curve, the variables time and time ${ }^{2}$ were included as predictors in all analyses, with lncort as dependent variable; addition of higher order polynomial terms did not improve model fit. The following confounders were examined in all models: gender; age; recent consumption of food or tobacco use (i.e. in the approximately $90 \mathrm{~min}$ interval since the previous ESM beep); use of oral contraceptives; recent awakening (samples taken within $60 \mathrm{~min}$ of awakening).

\section{Cortisol levels and diurnal slope}

To test whether mean cortisol level differed between sibling and control groups, a multi-level model was estimated with lncort as the dependent variable and the categorical variable group $(0=$ controls, $1=$ siblings) as independent variable. To examine whether familial vulnerability for psychosis was reflected in the diurnal cortisol slope, a group $\times$ time interaction term was added to the model.

\section{Cortisol reactivity to daily stressors}

To investigate whether stress elicited differential cortisol reactions in the two groups, a multi-level analysis was conducted with lncort as the dependent variable and group, event stress and their interaction as the independent variables.

Association between cortisol, psychotic experiences and negative affect

To examine whether increases in psychotic experiences or negative affect were associated with withinperson fluctuations in cortisol, we first conducted multi-level analyses with lncort as the dependent variable and group, psychotic experiences (and respectively negative affect) and their interaction as the independent variables. Next, negative affect and psychotic experiences and their interactions with group were included in the same model to determine whether negative affect might mediate the relationship between momentary psychotic experience and cortisol, given that negative emotions have been associated with both cortisol and psychosis (Myin-Germeys et al. 2001a; Freeman \& Garety, 2003; Thompson et al. 2007).

\section{Results}

\section{Compliance and sample characteristics}

Altogether, 14 participants (11 siblings and three control subjects) were unable to comply adequately with the research protocol (13 filled in fewer than 20 valid reports and one provided no saliva samples) and were therefore excluded from the analyses. The final sample included 123 participants (60 siblings and 63 controls), who completed a total of 5217 valid ESM observations. Control group participants completed significantly more ESM reports than the siblings [mean (s.D.) $=45.6$ (8.6) and 41.0 (9.3) reports, respectively; $F=8.31$, $p=0.01]$. Sample characteristics are summarized in Table 1. Sibling and control groups were well matched on demographic variables, but controls were on average 4.5 years older. With the exception of moderate differences in ESM compliance and age, the two groups did not differ significantly from each other on any of the displayed variables.

\section{Cortisol levels and diurnal slope}

Siblings had significantly higher cortisol levels over the ESM sampling moments than controls (Table 2). This pattern was present throughout the day, with no difference between the two groups in the steepness of the diurnal decline in cortisol secretion, as evidenced by the non-significant group $\times$ time of day effect shown in Table 2. 
Table 1. Demographic characteristics and descriptives

\begin{tabular}{|c|c|c|c|c|}
\hline & \multirow[b]{2}{*}{ Controls $(n=63)$} & \multirow[b]{2}{*}{ Siblings $(n=60)$} & \multicolumn{2}{|c|}{ Group comparisons } \\
\hline & & & Test statistic & $p$ value \\
\hline Age, years: mean (s.D.) & $33.27(10.3)$ & $28.82(10.0)$ & $F=5.93$ & 0.02 \\
\hline Gender (male:female) & $18: 45$ & $22: 38$ & $\chi^{2}(1)=0.98$ & 0.34 \\
\hline Education, $n(\%)^{\mathrm{a}}$ & & & $\chi^{2}(1)=2.53$ & 0.11 \\
\hline Secondary school or less & $20(31.8 \%)$ & $27(45.8 \%)$ & & \\
\hline Higher education & $43(68.3 \%)$ & $32(54.2 \%)$ & & \\
\hline Marital status, $n(\%)^{\mathrm{a}}$ & & & $\chi^{2}(1)=1.31$ & 0.25 \\
\hline Married or living together & $39(61.9 \%)$ & $31(51.7 \%)$ & & \\
\hline Never married/single/divorced & $24(38.2 \%)$ & $29(48.4 \%)$ & & \\
\hline Work situation, $n(\%)^{\mathrm{a}}$ & & & $\chi^{2}(1)=1.34$ & 0.51 \\
\hline Working/significant housework/studying & $61(98.8 \%)$ & $58(96.7 \%)$ & & \\
\hline Disabled or unemployed & $2(3.2 \%)$ & $2(3.3 \%)$ & & \\
\hline Living situation, $n(\%)^{\mathrm{a}}$ & & & $\chi^{2}(4)=7.85$ & 0.10 \\
\hline Alone & $7(11.1 \%)$ & $3(5 \%)$ & & \\
\hline With partner/family/children & $43(68.3 \%)$ & $33(55 \%)$ & & \\
\hline With parents/relatives & $10(15.9 \%)$ & $20(33.3 \%)$ & & \\
\hline Other & $3(4.8 \%)$ & $4(6.7 \%)$ & & \\
\hline CASH DSM-IV Axis I diagnosis lifetime, $\mathrm{n}(\%)^{\mathrm{a}}$ & & & $\chi^{2}(2)=1.56$ & 0.46 \\
\hline Depressive disorder, in partial remission & $3(4.8 \%)$ & $1(1.7 \%)$ & & \\
\hline Depressive disorder, in full remission & $8(12.7 \%)$ & $11(18.3 \%)$ & & \\
\hline No diagnosis & $52(82.5 \%)$ & $48(80 \%)$ & & \\
\hline CAPE trait score, mean (S.D.) & $1.0(0.14)$ & $1.1(0.21)$ & $F=2.04$ & 0.16 \\
\hline CTQ abuse, total score (S.D.) & $33.13(7.71)$ & $33.57(8.43)$ & $F=0.09$ & 0.76 \\
\hline Current smoker (no:yes) & $49: 14$ & $43: 17$ & $\chi^{2}(1)=0.61$ & 0.44 \\
\hline Current cannabis user (no:yes) & $61: 2$ & $57: 3$ & $\chi^{2}(1)=0.26$ & 0.61 \\
\hline \multicolumn{5}{|l|}{ ESM variables } \\
\hline Event stress, mean $^{\mathrm{b}}$ (S.D.) & 0.21 & 0.17 & $F=1.38$ & 0.24 \\
\hline Psychotic experiences, mean $^{\mathrm{b}}$ (S.D.) & $1.1(0.16)$ & $1.1(0.17)$ & $F=0.09$ & 0.78 \\
\hline Negative affect, mean ${ }^{\mathrm{b}}$ (S.D.) & $1.23(0.28)$ & $1.21(0.34)$ & $F=0.09$ & 0.76 \\
\hline Momentary cortisol, mean ${ }^{\mathrm{b}} \mathrm{nmol} / \mathrm{l}$ (s.D.) & $2.91(0.15)$ & $3.56(0.22)$ & $F=5.98$ & 0.02 \\
\hline
\end{tabular}

CASH, Comprehensive Assessment of Symptoms and History (Andreasen et al. 1992); CAPE, Community Assessment of Psychic Experiences; CTQ, Childhood Trauma Questionnaire; ESM, Experience Sampling Method.

${ }^{a}$ Due to rounding, percentages may not add exactly to $100 \%$.

${ }^{\mathrm{b}}$ For the experience sampling variables, an individual mean was first calculated over all reports; these values were then aggregated to obtain the group mean and s.D.

\section{Cortisol reactivity to daily stress}

Controls and siblings reported similar frequencies and intensities of daily negative events [mean (s.D.) $=14.5$ (12.3) and 13.1 (10), respectively; $F=0.49, p=0.49]$. There was no significant interaction between group and event stress in the model of negative affect $\left[\chi^{2}(1)=0.03, p=0.87\right]$, but a main effect of event stress $[\beta=0.11,95 \%$ confidence intervals (CI) $0.087-0.122$; $p<0.001]$. Multi-level analysis confirmed the main effect of group on cortisol $(\beta=0.17,95 \%$ CI 0.016-0.316, $p=0.03$ ) but revealed no main effect of event stress ( $\beta=0.01,95 \% \mathrm{CI}-0.013$ to $0.034 ; p=0.37)$. However, there was a significant interaction between group and event stress $\left[\chi^{2}(1)=4.11, p=0.04\right]$. Stratified analyses showed an increase in cortisol following unpleasant events in the siblings $(\beta=0.04,95 \%$ CI $0.003-0.075$, $p=0.03)$, but no such effect in the controls $(\beta=-0.01$, $95 \%$ CI -0.04 to $0.021 ; p=0.53$ ) (Fig. 1). Controlling for negative affect, a putative mediator of the stresscortisol relationship, had no substantial effect on the results [group $\times$ event stress interaction: $\chi^{2}(1)=4.71$, $p=0.03]$.

\section{Association between cortisol, psychotic experiences and negative affect}

There was no main effect of psychotic experiences on momentary cortisol. However, group moderated the effect of psychotic experiences on cortisol (Table 3, 
Table 2. Multi-level regression estimates for effects of group on cortisol level and diurnal slope

\begin{tabular}{lccrrr}
\hline & $\beta$ & $95 \%$ CI & S.E. & Z & $p$ \\
\hline Intercept & 1.137 & 0.780 to 1.494 & 0.182 & 6.25 & $<0.001$ \\
Group & 0.169 & 0.019 to 0.319 & 0.076 & 2.21 & 0.027 \\
Time of day (diurnal slope) & -0.130 & -0.134 to -0.125 & 0.002 & -54.43 & $<0.001$ \\
Group time of day & -0.003 & -0.009 to 0.004 & 0.003 & -0.85 & 0.393 \\
Age & -0.009 & -0.017 to -0.002 & 0.004 & -2.55 & 0.011 \\
Gender & -0.181 & -0.338 to -0.024 & 0.079 & -2.27 & 0.023 \\
Oral contraceptives & 0.155 & -0.015 to 0.325 & 0.087 & 1.79 & 0.074 \\
Recent awakening & 0.330 & 0.269 to 0.391 & 0.031 & 10.63 & $<0.001$ \\
Recent food intake & 0.161 & 0.128 to 0.193 & 0.016 & 9.77 & $<0.001$ \\
Recent smoking & 0.124 & 0.045 to 0.203 & 0.040 & 3.08 & 0.002 \\
\hline
\end{tabular}

The dependent variable is log-transformed cortisol (lncort). Regression coefficients are unstandardized. Group is coded 1 for siblings and 0 for controls. The variable time of day is centred around the grand mean. Time ${ }^{2}$ was also a significant predictor of lncort $(p<0.001)$ and is controlled for in all analyses. Recent awakening refers to samples taken within 60 min after awakening; recent food intake and smoking refer to reported occurrence of these activities in the interval between two Experience Sampling Method reports (roughly $90 \mathrm{~min}$ ).

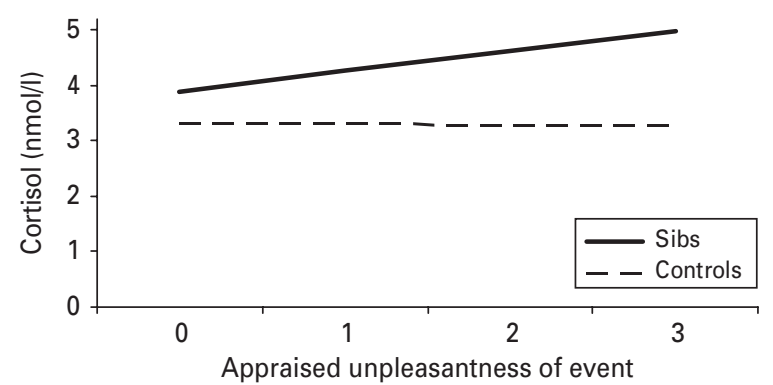

Fig. 1. Cortisol reactivity to stressful events. Modelled change (based on regression coefficient) in untransformed cortisol values (nmol/l) following daily events, according to their appraised unpleasantness $(0=$ neutral, $3=$ very unpleasant $)$, in sibling (sibs) and control groups.

Model 1). In the sibling group, increased levels of momentary psychotic experiences were associated with increased cortisol levels $(\beta=0.18,95 \%$ CI $0.081-$ $0.287 ; p<0.001)$, whereas in the control group, no association was found between psychotic experiences and cortisol $(\beta=-0.02,95 \% \mathrm{CI}-0.105$ to 0.075 ; $p=0.74)$. For negative affect, there was no main effect in the model predicting cortisol; however, an interaction was found with group (Table 3, Model 2). Stratified analyses indicated no significant association between negative affect and cortisol in the control group, whereas in the sibling group cortisol levels increased when participants reported higher negative affect. Finally, in a full model that included group, psychotic experiences, negative affect and the interactions between group and the two ESM variables, only the interaction between group and psychotic experiences remained significant (Table 3, Model 3).

\section{Post-hoc exploratory analyses}

To better understand the nature of the observed differences in cortisol patterns between sibling and control groups, we conducted a final series of multilevel analyses exploring the possible contribution of depression (presence or absence of lifetime diagnosis), childhood trauma (CTQ total score), overall current stress (mean unpleasantness of daily events during the sampling week) and distress (mean negative affect level) and degree of psychometric psychosis liability (CAPE trait score), by either exploring the interaction with group or adding the appropriate variable as additional predictor in the models of cortisol. Apart from some small effect size alterations, results were similar, with siblings having significantly higher cortisol levels over the ESM sampling moments and a significantly increased cortisol response following unpleasant events compared with controls. Some minor decrease of significance emerged only in the analyses controlling for psychometric psychosis liability [effect of group on cortisol: $\beta=0.15,95 \%$ CI -0.003 to $0.305 ; p=0.05$; interaction between group and event stress on cortisol: $\chi^{2}(1)=$ $3.49, p=0.06]$.

\section{Sensitivity analysis in non-depressed participants}

Additional analyses were carried out, investigating whether diagnoses of depression impacted the results. All analyses were repeated with exclusion of participants who had a current or past diagnosis of depression (controls $n=52$ and relatives $n=48$ remained in the analyses). The results remained the same. 
Table 3. Multi-level estimates of the effects of momentary psychotic experiences and negative affect on cortisol, as moderated by group (siblings v. controls)

\begin{tabular}{|c|c|c|c|}
\hline & \multicolumn{3}{|l|}{$\beta$ (S.E.) } \\
\hline & Model 1 & Model 2 & Model 3 \\
\hline Intercept & $1.12(0.19)^{* * *}$ & $1.09(0.18)^{* * *}$ & $1.10(0.19)^{* * *}$ \\
\hline Group & $-0.05(0.11)$ & $0.05(0.09)$ & $-0.08(0.11)$ \\
\hline Negative affect & & $0.02(0.03)$ & $0.02(0.03)$ \\
\hline Group $\times$ negative affect & & $0.10(0.04)^{* *}$ & $0.07(0.04)$ \\
\hline Psychotic experience & $-0.02(0.05)$ & & $-0.03(0.5)$ \\
\hline Group $\times$ psychotic experience & $0.20(0.07)^{* *}$ & & $0.15(0.08)^{*}$ \\
\hline
\end{tabular}

$\beta$, Unstandardized regression coefficient; S.E., standard error.

The dependent variable is lncort. The models control for time, time ${ }^{2}$, age, gender, oral contraceptive use, recent awakening, recent food intake and recent smoking.

${ }^{*} p<0.05,{ }^{* *} p<0.01,{ }^{* * *} p<0.001$.

\section{Discussion}

The current findings indicated that siblings of individuals with psychotic disorder had higher mean cortisol levels throughout the day and greater cortisol reactivity to daily stressors than a comparison group with no family history of psychosis. In the sibling but not the control group, rated intensities of momentary psychotic experiences and negative emotions were associated with transient increases in cortisol secretion. These findings suggest that alterations in HPA axis activity previously reported in patients with established psychotic disorder, unmedicated firstepisode patients, and ultra-high risk samples may also be present to some degree in healthy first-degree relatives.

The higher diurnal cortisol observed in the sibling group is consistent with reports of elevated HPA activity in patients with schizophrenia (Muck-Seler et al. 2004), first-episode psychosis (Ryan et al. 2004; Mondelli et al. 2010) and individuals at high risk for psychotic disorder (Mittal et al. 2007; Walker et al. 2010). However, other studies suggest a blunted cortisol response to stress in chronic and first episode psychosis (Jansen et al. 2000; van Venrooij et al. 2010). Together, these findings may support the notion that altered activity of the HPA axis may be a marker of underlying vulnerability for psychotic disorder. The finding of greater cortisol reactivity to daily stressors in siblings compared with controls provides new information, in light of the fact that previous studies of neuroendocrine responses to stress in psychotic disorder or at-risk samples were performed in the laboratory. In the control group, we expected to find relatively smaller cortisol stress reactivity to stress compared with the sibling group. In the current study, stressors were daily hassles and minor disturbances in the flow of everyday life. In comparison with experimentally induced stress, these were insufficiently stressful to influence cortisol secretion in our control group, without underlying vulnerability for psychotic disorder. The cortisol non-response in our control group may be a sign of good psychological health, with small daily events not significantly impacting on their stress axis. We have, however, no information about the immediate cortisol response after stressful events and can therefore not eliminate the possibility of an immediate but transient cortisol response with a short recovery time in the control group. Consistent with findings in ESM studies of increased emotional and psychotic symptom reactivity to minor daily stressors in relatives of patients with psychotic disorder (Myin-Germeys et al. 2005a,b), the current study reveals a similar pattern with regard to cortisol responses in the sibling group, supporting the idea that elevated biological stress sensitivity may be a vulnerability marker for psychosis. However, in contrast with the previous studies, the siblings did not differ from controls in their emotional reactivity to daily life stress. The current siblings may thus represent a relatively healthy subgroup with 'normal' levels of emotional stress reactivity, with increased cortisol response to daily hassles possibly reflecting a protective mechanism.

Another potentially important finding is that momentary increases in psychotic symptomatology were accompanied by increases in cortisol. This association between cortisol and momentary psychotic experiences can be interpreted in two causal directions. One plausible interpretation is that the distress associated with psychotic experiences and negative emotions could induce secondary increases in cortisol levels. Several studies have shown that momentary psychotic experiences are related to increases in negative affect 
(Myin-Germeys et al. 2001a ; Freeman \& Garety, 2003; Thewissen et al. 2010). Increases in momentary negative affect have, in turn, been linked to heightened cortisol secretion in healthy individuals (van Eck et al. 1996; Jacobs et al. 2007). In the current analyses, the estimated group $\times$ psychotic experiences interaction effect on cortisol decreased only slightly and remained significant after addition of negative affect and the group $\times$ negative affect interaction as predictors. In contrast, there was no significant association between negative affect and cortisol in this complete model. This suggests that subclinical psychotic experiences may have made a greater contribution than negative affect to increased cortisol levels in the sibling group. A second interpretation of the observed association between cortisol and momentary psychotic experiences reflects the possibility that increases in cortisol are either directly involved in the pathogenesis of psychotic experiences or reflect secondary, downstream processes. For instance, cortisol secretion increases dopamine activity in certain brain regions (Dallman et al. 2004). The dopaminergic system is thought to play an important role in the pathogenesis of positive psychotic symptoms (Laruelle \& AbiDargham, 1999). Thus, heightened cortisol secretion could theoretically, via dopaminergic pathways, increase the likelihood of clinical psychotic experiences. The current finding could be interpreted as evidence for a similar association with subclinical psychotic experiences in unaffected first-degree relatives, but whether this association is causal cannot be established in an ESM study. In general, the observed interrelationships among cortisol, psychotic experiences and negative emotions need to be further explored in longitudinal or experimental designs. Studies that either induce stress and measure subsequent changes in psychotic symptoms and emotions or that induce psychotic experience and subsequent changes in cortisol are needed to answer the question of causality more conclusively.

Dysregulation of the HPA axis is unlikely to be specific for psychosis, as it is found in many disorders, in particular major depression. Moreover, cortisol levels have been found to reflect depression and anxiety, but not psychotic symptoms, in a sample at ultra-high risk for psychosis (Thompson et al. 2007). Post-hoc analyses therefore examined whether a history of depression might explain the results; however, models that controlled for previous depressive episodes yielded similar findings. Alternatively, because siblings share not only genes but also the early rearing environment, childhood adversity - which has been associated with both psychotic disorder (Read et al. 2005) and HPA axis abnormalities (de Bellis et al. 1994; Tarullo \& Gunnar, 2006) - could also have influenced the current results. Although the two groups did not differ in self-reported childhood maltreatment, we also conducted post-hoc multi-level analyses to rule out the possibility that group differences in cortisol levels or reactivity were due to differential susceptibility to childhood abuse. These models revealed no association between abuse measures and cortisol in either of the two groups. Another set of analyses examined whether higher cortisol in the siblings might be due to greater overall current stress or distress. This was not the case. Finally, we found that overall psychometric psychosis liability did not significantly influence differences in cortisol patterns between control and sibling groups. Thus, in line with recent findings of cortisol abnormalities in first-episode psychosis, which could not be explained by stressful events, perceived stress or childhood trauma (Mondelli et al. 2010), the current results suggest that increased HPA axis activity may indeed reflect the underlying vulnerability to psychosis. The biological processes involved, however, remain to be elucidated.

This study has several limitations. First, use of ESM booklets instead of electronic devices means that the exact timing of participants' self-reports and saliva samples cannot be firmly established (Stone et al. 2002). However, results of a study comparing selfreported and electronically monitored saliva collection times, with the same intensive, semi-random timesampling protocol used in the current study, indicated that saliva was generally collected very close to the prescribed time and that self-reported collection times corresponded well with the electronic time stamps (Jacobs et al. 2005). Another comparative study concluded that paper and electronic diaries yield similar results (Green et al. 2006). Second, no saliva samples were taken at the time of awakening, so that the current dataset does not allow examination of the cortisol awakening response, a measure of HPA axis activity that appears to be blunted in first-episode psychosis (Mondelli et al. 2010). Third, this study focused solely on the circulating hormone cortisol and can thus provide no insight into underlying mechanisms at higher levels of the HPA axis. Fourth, the interval between cortisol sampling and event occurrence is relevant (van Eck et al. 1996). However, there is no reason to expect systematic differences between groups on the time interval between stressor and time of sampling. Therefore, we are confident that variability in time lag does not question the validity of our analysis on group differences. Moreover, a potentially stressful event could have occurred between 15 and $90 \mathrm{~min}$ before sampling of cortisol. As the half-life of cortisol is about $1 \mathrm{~h}$, we still expect to find traces of potential stressors, even when they occurred some time ago. Finally, caution is warranted in generalizing results 
from the current adult sibling sample to other groups at increased genetic risk for psychosis. Compared with children of parents with a psychotic disorder and others identified as being at ultra-high risk for developing a disorder, the siblings included in this study were older; in most cases, past the age when onset of psychosis is most likely to occur. The nature and extent of HPA axis abnormalities in healthy siblings may differ from those described in at-risk and ultra-high risk adolescent samples. Therefore, it cannot be eliminated that the increased cortisol reactivity in our sibling group reflects a protective rather than a risk factor. On the other hand, it might be that even with a suboptimal biological reactivity, siblings manage to cope better with stress and/or have other resilience factors protecting them from psychotic disorder.

The current study also had some specific strengths. In particular, the repeated sampling of salivary cortisol over 6 days takes into account the well-known but often ignored unreliability of cortisol measures obtained at infrequent intervals (Hruschka et al. 2005). Multiple cortisol measures per person were complemented by a relatively large number of participants. Use of multi-level modelling allowed assessment of within-person associations between cortisol and subjective experience in real time and real-life contexts. Although cortisol measures in both groups were within the normal range, intensive sampling revealed a consistent pattern of heightened HPA activity in the siblings. Taken together, the current findings lend additional credence to the hypothesis that irregularities in HPA axis activity are involved in psychosis vulnerability, broadening the spectrum of vulnerability to include siblings without demonstrable psychometric risk markers.

\section{Appendix. Genetic Risk and Outcome in Psychosis (G.R.O.U.P.) investigators}

R. S. Kahn, D. H. Linszen, J. van Os, D. Wiersma, R. Bruggeman, W. Cahn, L. de Haan, L. Krabbendam, I. Myin-Germeys.

\section{Acknowledgements}

Inez Myin-Germeys was supported by a 2006 NARSAD Young Investigator award and by the Dutch Medical Council (VENI and VIDI grant). This work was also sponsored by the Dutch Organization for Scientific Research NWO (G.R.O.U.P.). The authors thank research assistants Truda Driesen and Frieda Goethem for their work on this project.

\section{Declaration of Interest}

None.

\section{References}

Albus M, Ackenheil M, Engel RR, Muller F (1982). Situational reactivity of autonomic functions in schizophrenic patients. Psychiatry Research 6, 361-370.

Andreasen NC, Flaum M, Arndt S (1992). The Comprehensive Assessment of Symptoms and History (CASH). An instrument for assessing diagnosis and psychopathology. Archives of General Psychiatry 49, 615-623.

APA (1994). Diagnostic and Statistical Manual of Mental Disorders, fourth edition. American Psychiatric Association: Washington, DC.

Bernstein DP, Stein JA, Newcomb MD, Walker E, Pogge D, Ahluvalia T, Stokes J, Handelsman L, Medrano M, Desmond D, Zule W (2003). Development and validation of a brief screening version of the Childhood Trauma Questionnaire. Child Abuse \& Neglect 27, 169-190.

Breier A, Wolkowitz OM, Doran AR, Bellar S, Pickar D (1988). Neurobiological effects of lumbar puncture stress in psychiatric patients and healthy volunteers. Psychiatry Research 25, 187-194.

Brenner K, Liu A, Laplante DP, Lupien S, Pruessner JC, Ciampi A, Joober R, King S (2009). Cortisol response to a psychosocial stressor in schizophrenia: blunted, delayed, or normal? Psychoneuroendocrinology 34, 859-868.

Csikszentmihalyi M, Larson R (1987). Validity and reliability of the Experience-Sampling Method. Journal of Nervous and Mental Disease 175, 526-536.

Dallman MF, Akana SF, Strack AM, Scribner KS, Pecoraro N, La Fleur SE, Houshyar H, Gomez F (2004). Chronic stress-induced effects of corticosterone on brain: direct and indirect. Annals of the New York Academy of Sciences 1018, 141-150.

de Bellis MD, Chrousos GP, Dorn LD, Burke L, Helmers K, Kling MA, Trickett PK, Putnam FW (1994).

Hypothalamic-pituitary-adrenal axis dysregulation in sexually abused girls. Journal of Clinical Endocrinology $\mathcal{E}$ Metabolism 78, 249-255.

Delespaul P, deVries M, van Os J (2002). Determinants of occurrence and recovery from hallucinations in daily life. Social Psychiatry and Psychiatric Epidemiology 37, 97-104.

Freeman D, Garety PA (2003). Connecting neurosis and psychosis : the direct influence of emotion on delusions and hallucinations. Behavioral Research and Therapy 41, 923-947.

Garner B, Pariante CM, Wood SJ, Velakoulis D, Phillips L, Soulsby B, Brewer WJ, Smith DJ, Dazzan P, Berger GE, Yung AR, van den Buuse M, Murray R, McGorry PD, Pantelis C (2005). Pituitary volume predicts future transition to psychosis in individuals at ultra-high risk of developing psychosis. Biological Psychiatry 58, 417-423.

Goldstein JM, Seidman LJ, Makris N, Ahern T, O'Brien LM, Caviness Jr. VS, Kennedy DN, Faraone SV, Tsuang MT (2007). Hypothalamic abnormalities in schizophrenia: sex effects and genetic vulnerability. Biological Psychiatry 61, 935-945.

Green AS, Rafaeli E, Bolger N, Shrout PE, Reis HT (2006). Paper or plastic? Data equivalence in paper and electronic diaries. Psychological Methods 11, 87-105.

G.R.O.U.P. (2010). Evidence that familial liability for psychosis is expressed as differential sensitivity to 
cannabis: an analysis of patient-sibling and sibling-control pairs. Archives of General Psychiatry 68, 138-147.

Hanssen M, Bak M, Bij1 R, Vollebergh W, van Os J (2005). The incidence and outcome of subclinical psychotic experiences in the general population. British Journal of Clinical Psychology 44, 181-191.

Hruschka DJ, Kohrt BA, Worthman CM (2005). Estimating between- and within-individual variation in cortisol levels using multilevel models. Psychoneuroendocrinology 30, 698-714.

Jacobs N, Myin-Germeys I, Derom C, Delespaul P, van Os J, Nicolson NA (2007). A momentary assessment study of the relationship between affective and adrenocortical stress responses in daily life. Biological Psychology 74, 60-66.

Jacobs N, Nicolson NA, Derom C, Delespaul P, van Os J, Myin-Germeys I (2005). Electronic monitoring of salivary cortisol sampling compliance in daily life. Life Sciences 76, 2431-2443.

Jansen LM, Gispen-de Wied CC, Gademan PJ, De Jonge RC, van der Linden JA, Kahn RS (1998). Blunted cortisol response to a psychosocial stressor in schizophrenia. Schizophrenia Research 33, 87-94.

Jansen LM, Gispen-de Wied CC, Kahn RS (2000). Selective impairments in the stress response in schizophrenic patients. Psychopharmacology (Berlin) 149, 319-325.

Koolschijn PC, van Haren NE, Hulshoff Pol HE, Kahn RS (2008). Hypothalamus volume in twin pairs discordant for schizophrenia. European Neuropsychopharmacology 18, 312-315.

Laruelle M, Abi-Dargham A (1999). Dopamine as the wind of the psychotic fire: new evidence from brain imaging studies. Journal of Psychopharmacology 13, 358-371.

Marcelis M, Cavalier E, Gielen J, Delespaul P, van Os J (2004). Abnormal response to metabolic stress in schizophrenia: marker of vulnerability or acquired sensitization? Psychological Medicine 34, 1103-1111.

Meltzer HY, Koenig JI, Nash JF, Gudelsky GA (1989). Melperone and clozapine: neuroendocrine effects of atypical neuroleptic drugs. Acta Psychiatrica Scandinavica (Suppl.) 352, 24-29.

Mittal VA, Dhruv S, Tessner KD, Walder DJ, Walker EF (2007). The relations among putative biorisk markers in schizotypal adolescents: minor physical anomalies, movement abnormalities, and salivary cortisol. Biological Psychiatry 61, 1179-1186.

Mondelli V, Dazzan P, Gabilondo A, Tournikioti K, Walshe M, Marshall N, Schulze KK, Murray RM, McDonald C, Pariante CM (2008). Pituitary volume in unaffected relatives of patients with schizophrenia and bipolar disorder. Psychoneuroendocrinology 33, 1004-1012.

Mondelli V, Dazzan P, Hepgul N, di Forti M, Aas M, d'Albenzio A, di Nicola M, Fisher H, Handley R, Marques TR, Morgan C, Navari S, Taylor H, Papadopoulos A, Aitchison KJ, Murray RM, Pariante CM (2010). Abnormal cortisol levels during the day and cortisol awakening response in first-episode psychosis: the role of stress and of antipsychotic treatment. Schizophrenia Research 116, 234-242.

Muck-Seler D, Pivac N, Mustapic M, Crncevic Z, Jakovljevic M, Sagud M (2004). Platelet serotonin and plasma prolactin and cortisol in healthy, depressed and schizophrenic women. Psychiatry Research 127, 217-226.

Myin-Germeys I, Delespaul P, van Os J (2005a). Behavioural sensitization to daily life stress in psychosis. Psychological Medicine 35, 733-741.

Myin-Germeys I, Marcelis M, Krabbendam L, Delespaul P, van Os J (2005b). Subtle fluctuations in psychotic phenomena as functional states of abnormal dopamine reactivity in individuals at risk. Biological Psychiatry 58, 105-110.

Myin-Germeys I, Nicolson NA, Delespaul PA (2001a). The context of delusional experiences in the daily life of patients with schizophrenia. Psychological Medicine 31, 489-498.

Myin-Germeys I, Oorschot M, Collip D, Lataster J, Delespaul P, van Os J (2009). Experience sampling research in psychopathology: opening the black box of daily life. Psychological Medicine 39, 1533-1547.

Myin-Germeys I, van Os J, Schwartz JE, Stone AA, Delespaul PA (2001b). Emotional reactivity to daily life stress in psychosis. Archives of General Psychiatry 58, 1137-1144.

Nicolson N (2007). Measurement of cortisol. In Handbook of Physiological Research Methods in Health Psychology (eds. L. J. Luecken and L. C. Gallo), pp. 37-74. Sage Publications: Thousand Oaks, CA.

NIMH Genetics Initiative (1992). Family Interview for Genetic Studies (FIGS). National Institute of Mental Health: Rockville, MD.

Pariante CM (2008). Pituitary volume in psychosis: the first review of the evidence. Journal of Psychopharmacology 22, 76-81.

Peeters F, Nicolson NA, Berkhof J (2004). Levels and variability of daily life cortisol secretion in major depression. Psychiatry Research 126, 1-13.

Read J, van Os J, Morrison AP, Ross CA (2005). Childhood trauma, psychosis and schizophrenia: a literature review with theoretical and clinical implications. Acta Psychiatrica Scandinavica 112, 330-350.

Ryan MC, Sharifi N, Condren R, Thakore JH (2004). Evidence of basal pituitary-adrenal overactivity in first episode, drug naive patients with schizophrenia. Psychoneuroendocrinology 29, 1065-1070.

Soliman A, O'Driscoll GA, Pruessner J, Holahan AL, Boileau I, Gagnon D, Dagher A (2008). Stress-induced dopamine release in humans at risk of psychosis: a [11C]raclopride PET study. Neuropsychopharmacology 33, 2033-2041.

StataCorp (2009). STATA/SE Statistical Software, Release 11. StataCorp: College Station, TX.

Stone AA, Shiffman S, Schwartz JE, Broderick JE, Hufford MR (2002). Patient non-compliance with paper diaries. British Medical Journal 324, 1193-1194.

Sulon J, Demey-Ponsart L, Beauduin P, Sodoyez JC (1978). Radioimmunoassay of corticosterone, cortisol and cortisone: their application to human cord and maternal plasma. Journal of Steroid Biochemistry and Molecular Biology 9, 671-676.

Tarullo AR, Gunnar MR (2006). Child maltreatment and the developing HPA axis. Hormones and Behavior 50, 632-639. 
Thewissen V, Bentall RP, Oorschot M, Campo JA, van Lierop T, van Os J, Myin-Germeys I (2010). Emotions, selfesteem, and paranoid episodes: an experience sampling study. British Journal of Clinical Psychology, doi: 10.1348/ 014466510X508677, published online 2 March 2011.

Thompson KN, Phillips LJ, Komesaroff P, Yuen HP, Wood SJ, Pantelis C, Velakoulis D, Yung AR, McGorry PD (2007). Stress and HPA-axis functioning in young people at ultra high risk for psychosis. Journal of Psychiatric Research 41, 561-569.

van Eck M, Berkhof H, Nicolson N, Sulon J (1996). The effects of perceived stress, traits, mood states, and stressful daily events on salivary cortisol. Psychosomatic Medicine 58, 447-458.

van Venrooij JA, Fluitman SB, Lijmer JG, Kavelaars A, Heijnen CJ, Westenberg HG, Kahn RS,

Gispen-de Wied CC (2010). Impaired neuroendocrine and immune response to acute stress in medication-naive patients with a first episode of psychosis. Schizophrenia Bulletin, doi : 10.1093/schbul/sbq062, published online 17 June 2010.

van Winkel R, Stefanis NC, Myin-Germeys I (2008). Psychosocial stress and psychosis. A review of the neurobiological mechanisms and the evidence for gene-stress interaction. Schizophrenia Bulletin 34, 1095-1105.

Walker E, Mittal V, Tessner K (2008). Stress and the hypothalamic pituitary adrenal axis in the developmental course of schizophrenia. Annual Review of Clinical Psychology 4, 189-216.

Walker EF, Brennan PA, Esterberg M, Brasfield J, Pearce B, Compton MT (2010). Longitudinal changes in cortisol secretion and conversion to psychosis in at-risk youth. Journal of Abnormal Psychology 119, 401-408.

Walker EF, Walder DJ, Reynolds F (2001). Developmental changes in cortisol secretion in normal and at-risk youth. Development and Psychopathology 13, 721-732.

Walsh P, Spelman L, Sharifi N, Thakore JH (2005). Male patients with paranoid schizophrenia have greater ACTH and cortisol secretion in response to metoclopramideinduced AVP release. Psychoneuroendocrinology 30, 431-437.

Wik G (1995). Effects of neuroleptic treatment on cortisol and 3-methoxy-4-hydroxyphenylethyl glycol levels in blood. Journal of Endocrinology 144, 425-429.

Wright IC, Rabe-Hesketh S, Woodruff PW, David AS, Murray RM, Bullmore ET (2000). Meta-analysis of regional brain volumes in schizophrenia. American Journal of Psychiatry 157, 16-25. 